\title{
Cognitive Capacity Genomewide Polygenic Scores Identify Individuals Resilient to Cognitive Decline in Aging
}

Running title: Polygenic underpinning of cognitive performances and their declines

Yoonjung Yoonie Joo, Ph.D ${ }^{1,}$, Jiook Cha, Ph.D ${ }^{2,3,4}$, Jeremy Freese, Ph.D5 , M Geoffrey Hayes, Ph. $D^{1,6,7}$.

1. Division of Endocrinology, Metabolism, and Molecular Medicine, Department of Medicine, Northwestern University Feinberg School of Medicine, Chicago, IL, 60611, USA

2. Department of Psychology, College of Social Sciences, Seoul National University, Seoul, 08825, South Korea

3. Department of Brain and Cognitive Sciences, College of Natural Sciences, Seoul National University, Seoul, 08825, South Korea

4. Al Institute, Seoul National University, Seoul, 08825, South Korea

5. Department of Sociology, School of Humanities and Sciences, Stanford University, Palo Alto, CA, 94305, USA

6. Center for Genetic Medicine, Northwestern University Feinberg School of Medicine, Chicago, IL, 60611, USA

7. Department of Anthropology, Northwestern University, Evanston, IL, 60208, USA

8. * Current Affiliation: Department of Psychology, College of Social Sciences, Seoul National University, Seoul, 08825, South Korea

\section{Correspondence}

M. Geoffrey Hayes

Division of Endocrinology, Metabolism, and Molecular Medicine

Department of Medicine,

Northwestern University Feinberg School of Medicine

Chicago, IL 60611

Email:ghayes@northwestern.edu 
Phone: $312-503-4141$

Fax: 312-908-9032

\section{Disclosures}

The authors have no conflicts to declare.

\section{Data Availability}

The data/analyses presented in the current publication have been deposited in and are available from the dbGaP database under dbGaP accession phs001157.v1.p1.

\section{Keywords}

Sociogenomics, Phenome-wide association study, Polygenic risk score, Aging genetics, Cognitive genetics

Word counts

2,776 (not including abstract, online methods, references and figure legends) 


\section{Abstract}

The genetic underpinnings of cognitive resilience in aging remains unknown. Predicting an individual's rate of cognitive decline-or cognitive resilience-using genetics will allow personalized intervention for cognitive enhancement and optimal selection of target samples in clinical trials. Here, using genome-wide polygenic scores(GPS) as the genomic indicators for variations of human intelligence, we examined the genetic liability of cognitive abilities in the behavioral/cognitive phenome to understand individual differences in cognitive capacity over time. Using the longitudinal sociogenomic data of 8,509 European-ancestry adults between the ages of mid-60s to 70 s, we found that a higher cognitive GPS significantly correlated with a slower cognitive decline specifically in memory recall, but not in other cognitive domains. Linear mixed models with cognitive GPSs explained proportions of the variances in cognitive tests up to $60.4 \%$. This study presents the novel genetic protective effects of cognitive ability on the decline of memory recall in aging population. 


\section{Introduction}

The magnitudes of cognitive decline in aging, a major health concern in contemporary society, differ substantially across individuals ${ }^{1,2}$. Unraveling the genetic underpinning for individual variations of cognitive decline with aging, particularly those associated with cognitive resilience, could help develop individualized interventions for cognitive decline and allow better sample selection in clinical trials in dementia research. Despite studies reporting the genetic risk factors of accelerated cognitive decline among individuals with dementia ${ }^{3-5}$, we know little about the genetic protective factors of cognitive resilience in the normal aging population. Genomewide Polygenic Scores (GPS) leverage the fact that most human traits are developed from the aggregated influence of many genetic variants, both common and rare ${ }^{6-8}$. By aggregating the miniscule effects of millions of genetic variants into a single score, GPS allows researchers to stratify individuals by their genomic propensity for a particular trait and to select individuals with extremely high or low GPS for further research. The recent large genome-wide association studies (GWAS) of educational attainment, a well-established proxy phenotype to human intelligence, identified 1,271 independent autosomal loci reaching 17 genome-wide significance ${ }^{9}$. These findings suggest several biological pathways related to brain development or neuron-to-neuron communication contribute to human intelligence. While the GWAS revealed many genetic variants associated with cognitive phenotypes (such as cognitive performance, math ability, highest math class taken) ${ }^{9-16}$, the genomic contribution to specific cognitive domains remains unknown, as does their relationship to cognitive changes with aging.

Since general cognitive ability is known to be highly heritable (50-70\%) and polygenic ${ }^{17,18} 12,17$, we utilize GPS to account for the genome-wide factors underlying cognitive capacity and its secular changes. We leverage the expansive phenotype information of a $50_{+}$ year social longitudinal database for phenomewide association studies (PheWAS). The Wisconsin Longitudinal Study (WLS), the longest-running social longitudinal study in the United States ${ }^{19,20}$, encompasses a detailed and broad lifelog of cognition, personality, financial, health, and socioeconomic status. The sample is based on 10,317 individuals surveyed in

301957 - representing a 1/3 random sample of Wisconsin high school graduates that year - with 
32 same cognitive ability tests with the time interval of $\sim 10$ years in their latest survey rounds, as 33 well as collected the genotype data of the participants, which creates a deep genotype-

34 phenotype catalogue of an individual's cognitive and behavioral traits over their adult lives.

Herein, we hypothesize that the polygenic influence of the cognitive phenotypes can explain certain patterns of cognitive abilities and their declines in aging as well as other sociobehavioral phenotypes that might be affected by genetics of cognitive phenotypes. We tested the associations between longitudinal observations of individual cognitive/behavioral phenomes and the GPSs of four different cognitive phenotypes (educational attainment, cognitive performance, math ability, highest math class taken) with a focus on the secular changes of cognitive test scores. There are three important aspects in this approach: firstly, whether a certain cognitive domain is more impacted by polygenic influence than other cognitive domains; secondly, whether individuals with different GPS show different patterns of cognitive decline in aging; and, thirdly, the extents to which phenotypic variances of behavioral/cognitive phenotypes can be explained by genetic liability of cognitive capacities.

\section{Results}

Cognitive GPS correlates with the changes in delayed recall and immediate recall

The analysis sample includes 8,509 European-ancestry individuals, and cognitive assessments included 6 different cognitive ability tests. Among the 6 cognitive performance tests, the WLS had administered items from the WAIS Similarities ${ }^{21}$ subtest three times over the survey period and the others twice (Immediate and Delayed Recall, Letter and Category Fluency, and Digit Ordering). The first cognitive assessments were conducted in the average age of 64-65 for original WLS participants (WLS survey round 5, 2003-2004), and the subsequent assessment in the age of 71 (WLS survey round 6,2011 ). To capture the polygenic nature of general cognitive ability, a set of cognitive ability-related GPSs were constructed based on four large-scale GWASs on educational attainment ( $E A, n=1,131,881$ ), cognitive performance ( $C P, n=257,841$ ), self-reported math ability (MA, $n=564,698$ ), and highest-level math class taken $(\mathrm{HM}, \mathrm{n}=430,445)$ from Lee et $\mathrm{l}^{9}{ }^{9}$. We used linear mixed model with interaction terms to test whether the four cognitive GPSs can quantify the inherited 
63 portions of secular cognitive test scores changes which were recorded repetitively with an 8-964 year interval. Among those GPSs, two representative cognitive GPSs of EA and CP are reported throughout the main text, and the results for MA/HM GPSs are available in

\section{Supplementary Material.}

Results showed that higher GPS for cognitive phenotypes were significantly associated with the changes of Delayed Recall ( $p$-value $=1.82 \mathrm{E}-07$ with CP GPS) and Immediate Recall test scores ( $p$-value=3.23E-10 with CP GPS) after accounting for interaction effects with time. The patterns were consistent across all four cognitive GPSs that have been examined on Delayed and Immediate Recall tests. Compared to individuals with 1 SD standard deviation (SD) higher EA GPS (GPS mean=-0.131), individuals with 1SD lower GPS (GPS mean=0.439) showed stronger associations in Delayed Recall score decline in their later survey round ( $\beta=-3.69 \mathrm{E}-03$ versus $-4.77 \mathrm{E}-02$ ). Interestingly, significant increases were observed for Immediate Recall test scores among the individuals with high cognitive GPS. Individuals with 1SD higher GPS for cognitive phenotypes showed increased performance in the Immediate Recall test over time ( $\beta=2.50 \mathrm{E}-02, \mathrm{SE}=5.28 \mathrm{E}-03$ ) whereas individuals with $1 \mathrm{SD}$ lower GPS for cognitive phenotypes showed decreased performance in the same test $(\beta=-2.19 \mathrm{E}-02$, $\mathrm{SE}=5.28 \mathrm{E}-03$ ) (Table 1, Figure 1).

Similarities task most strongly correlates with cognitive GPS

Without a time interaction term, the secular changes observed in the Similarities test score marked the strongest $p$-value significance $(p$-value $=1.20 \mathrm{E}-117, \beta=0.46)$ with cognitive GPS, when assuming random variances for each participant through the linear mixed model. For individual assessments of the Similarities task, the effect size ( $\beta$ ) of GPS stayed robust, ranging from 1.26 (R5) to 1.36 (R6) (Figure 2(a) and Figure 3(a)). Other cognitive decline phenotypes, such as changes in Digit Ordering ( $\beta=0.33$, p-value $=1.00 \mathrm{E}-51,95 \% \mathrm{Cl}=(0.29$, 0.37)), Category Fluency $(\beta=0.25$, $p$-value $=8.99 E-46,95 \% \mathrm{Cl}=(0.24,0.31))$ and Letter Fluency $(\beta=0.27, p$-value $=2.24 \mathrm{E}-34,95 \% \mathrm{Cl}=(0.23,0.32))$ also presented strong associations with $\mathrm{EA}$ show less changes in cognitive phenotypes over time. 
Polygenic Effect of Educational Attainment in the Cognitive Phenome (EA GPS

94 PheWAS)

\section{Polygenic Effect of Cognitive Performance in the Cognitive Phenome (CP GPS PheWAS)}

$$
\text { Across all of our PheWAS, the association between IQ score and CP GPS had the }
$$

strongest significance in terms of $p$-value and the variance explained $\left(R^{2}\right)(\beta=1.48, p$ value $=1.12 \mathrm{E}-196,95 \% \mathrm{Cl}=(1.38,1.57)$ ) (Figure 2(b), 3(b) and Table 2). The variance of $\mathrm{IQ}$ scores explained by CP GPS was $11.5 \%$ (Adjusted $\mathrm{R}^{2} 11.4 \%$ ), whereas $9.8 \%$ (Adjusted $\mathrm{R}^{2}$ $9.6 \%$ ) of IQ variance was explained by EA GPS (Table 2).

The PheWAS of CP GPS in the cognitive phenome largely showed a similar pattern to the EA GPS PheWAS. Several cognitive tests were consistently and firmly associated with CP GPS: Similarities (R4, $\beta=0.87$, $p$-value $=2.48 E-65,95 \% \mathrm{Cl}=(0.77,0.97)$ ), Number Series 
$124(\beta=0.67, p$-value $=8.14 \mathrm{E}-73,95 \% \mathrm{Cl}=(0.57,0.77))$, 'digit ordering' $(\mathrm{R} 6, \beta=0.39, \mathrm{p}$-value $=1.16 \mathrm{E}-$ $12539,95 \% \mathrm{Cl}=(0.29,0.49))$, and Letter Fluency $(\mathrm{R} 6, \beta=0.60, p$-value $=1.36 \mathrm{E}-28,95 \% \mathrm{Cl}=(0.50$, 126 0.71)). Among the cognitive decline phenotypes, changes in the Similarities task marked the 127 best $p$-value association ( $\beta=0.29$, $p$-value $=3.00 \mathrm{E}-100$ ), followed by the changes in Digit 128 Ordering ( $\beta=0.28$, $p$-value $=7.33 E-76)$. We also confirmed the strong associations of CP GPS 129 with high school class rank ( $\beta=1.24$, $p$-value $=8.23 \mathrm{E}-87,95 \% \mathrm{Cl}=(1.12,1.36)$ ), the years of 130 educational attainment $(\beta=0.79$, $p$-value $=5.72 E-74,95 \% \mathrm{Cl}=(0.70,0.89))$, occupational 131 education score $(\beta=0.55$, $\mathrm{p}$-value $=4.12 \mathrm{E}-35,95 \% \mathrm{Cl}=(0.46,0.65))$ and occupational income 132 score $(\beta=0.45$, $p$-value $=3.59 \mathrm{E}-20,95 \% \mathrm{Cl}=(0.35,0.55))$ as we observed in EA GPS PheWAS

133 (Figure 2(b) and Supplementary Table).

\section{Discussion} cognitive phenome using a combinational approach of GPS-based PheWAS on longitudinal observations of cognitive assessments. Our mixed-effect linear model examined the role of cognitive GPS on the changes of cognitive capacity in aging, and additional analyses tested the phenomewide associations between cognitive GPS and several cognitive/behavioral traits. In conjunction with genotype data, the availability of cognitive phenome data from the WLS enabled exploration of cognitive/behavioral phenotypes with longitudinal measurements of human cognitive ability.

In addition to the wide range of the cognitive domains correlating with the cognitive GPS, a decline of memory recall in aging correlated with the cognitive GPS but not the other cognitive domains did. Memory recall, assessed by immediate and delayed recall tests of words, is hippocampus dependent ${ }^{22-24}$. It is interesting that the discovered genetic protective effect exerted specifically on the hippocampus-related memory recall. There are two implications worth noting. Firstly, given the specificity of the correlations among various cognitive domains, the genetic protective factor of memory decline may be mediated via the hippocampus. Indeed, the hippocampus is the primary mediator of interventions for the cognitive wellness or dementia, such as aerobic fitness ${ }^{25}$, diet ${ }^{26}$, and medication ${ }^{27-29}$. This is 
155

closely related to the unique role of the hippocampus in neurogenesis and synaptic plasticity ${ }^{30,31}$. Future research should thus test whether the hippocampus and hippocampal network underlies the genetic projective effect on memory decline, and if so seek to elucidate the mechanisms involved. Secondly, given the role of the hippocampal memory impairment in the pathophysiology of Alzheimer's disease (AD), our finding may lead to the potential link of the inherited genetic factor of cognitive resilience to the individual differences in hippocampal degeneration, as well as memory decline in $A D^{32,33}$. Testing this link will allow better stratification of $A D$ and monitoring the course of the disease by the individual-specific genetic profiles of cognitive resilience.

Considering that the correlations of the time-x-GPS interactions were positive (Table 1), individuals with a higher cognitive GPS presented a slower trajectory of memory decline than those with a lower GPS. This result indicates that the portions of cognitive ability under genetic influence may serve as a 'buffer' against memory decline in aging. These observations align well with existing studies on the protective effect of education and intelligence on the occurrence of dementia ${ }^{34}$. A close relationship between early-life education and intelligence with cognitive decline have been reported for dementia and $A D^{35}$. Even though it is not yet clear how early-life education and intelligence moderate the risk for dementia, our findings suggest that individual variations of memory decline are closely associated with polygenic influences of cognitive abilities.

Among the WLS cognitive modules, the Similarities task from WAIS and the Number Series task showed the strongest associations across the four cognitive GPSs in terms of effect size $(\beta)$ and $p$-value, indicating its high genetic relatedness. These findings suggest that the cognitive components required to successfully complete the Similarities or Number Series tasks might strongly overlap with genetic components of cognitive abilities primarily exhibited by the domain of fluid intelligence. All the Similarities tasks performed in three different time points (R4, R5, R6) presented significant PheWAS associations following other early-life cognition measures, such as IQ scores and high school academic achievement, suggesting its high relatedness to early-life cognition. The effect sizes of the Similarities tasks were fairly consistent and robust over time (R4: 1.26, R5: 1.36, R6: 1.36 with EA GPS).

Fluid intelligence is used to think and act flexibly and quickly, and to encode new episodic memories to solve problems in novel situations without previously existing knowledge. 
186 The series of cognitive components involved in the Similarities and Number series tasks, such 187 as logical memory, symbol search, and reasoning, might be closely linked to early-life 188 cognition, all of which may serve as phenomewide indicators for fluid intelligence. Our findings 189 are backed up by the previous knowledge that fluid intelligence is considered to be more 190 dependent on biological influences and less dependent on past learning experiences than 191 crystalized intelligence ${ }^{36}$.

192 The IQ scores of individuals were best predicted by CP GPS in terms of both $p$-value 193 and effect size (Figure 2, 3). The strong genetic association between cognitive ability and 194 educational attainment has been well established in several GWAS studies on human 195 intelligence ${ }^{9,13-16}$. IQ scores of the WLS respondents were derived from the Henmon-Nelson 196 test of mental ability, which was administered to high school students in Wisconsin in the 197 1950s. The test is regarded as a general measure of overall intelligence, capturing both fluid 198 and crystallized intelligence. We found the close associations between IQ score and cognitive 199 GPS as well as its high correlations to several early-life academic achievement such as high 200 school rank. (Figure 2) Those findings jointly showed that substantial variances of IQ scores 201 could be explained by several intercorrelated phenotypes in both domains of cognitive ability 202 and educational achievements.

Another interesting aspect of our study was the systematic examination of the relationships between cognitive GPS and behavioral phenotypes. Our PheWAS of the 206 from cognitive abilities. Several cognitive GPSs significantly correlated with Openness among 207 the Big 5 personality factors, but not with others. Another personality trait, Neuroticism, 208 showed a significant negative correlation with the same range of cognitive GPS ${ }^{37}$. The finding 209 presents an interesting cross-trait hypothesis in which the variances of cognitive abilities may 210 be partially explained by a personality dimension. 'Openness' could be regarded as the attitude 211 and tendency to explore, detect, understand, and appreciate complicated patterns of new 212 information through both the senses and in the abstract ${ }^{38}$. Previous studies support our 213 findings, concluding that an overall open-minded attitude might positively influence the long214 term variances of cognitive abilities with willingness to explore ${ }^{39}$.

215 No significant associations between Spouse IQ and cognitive abilities were confirmed, 216 which indicates that the behavioral associations between assortative mating and cognitive 
217 abilities is unclear. In addition, a strong relationship between occupational income and several 218 cognitive GPS were found, which supports the existing studies on a strong association 219 between general mental ability and job performance ${ }^{40}$.

220 The GPS-based PheWAS found that up to $11.5 \%$ (adjusted $R^{2} 11.4 \%$ ) of variances in 221 IQ score could be explained with CP GPS. The variances in academic achievement in high 222 school were explained as high as $6.2-9.2 \%$ across GPS of cognitive abilities. Among the WLS 223 cognitive test modules, up to $4.8 \%$ of the Similarities task score variances was explained by 224 EA GPS (Table 2). Those findings demonstrate that a substantial portion of phenotypic variances in several cognitive abilities could be explained and influenced by intelligence genetic risk variants, presented as several GPS.

Several limitations of this study should be noted. The WLS had two time points for measuring changes in their cognitive assessments with an 8-9 year interval. Adding more cognitive measurements through time will strengthen our findings by more thoroughly monitoring cognitive changes over the lifetime. Also, the unexplored impact of other sociodemographic variables such as socioeconomic status, educational environment, lifestyles, or family structure, should be considered to better connect our theorical findings with phenome-wide expression of cognitive abilities. In addition, we used European-ancestry specific summary statistics for constructing the cognitive GPSs and applied them to participants with European-ancestry. Researchers should note that the translational application to non-European individuals could be different and the results should be interpreted with caution. Future investigation is needed to elucidate heterogeneity between ancestry groups for the genetic underpinnings of cognitive abilities.

Our analytic approach combining GPS and PheWAS leverages the fact that human intelligence has a considerably polygenic and pleiotropic architecture in its phenotypic expression ${ }^{12,17,41}$. Through this study, we confirmed potential utility of GPS on future prediction of dementia or other neurocognitive disorders. Our findings could serve as the first cognitivephenome map that describes the functional boundaries of human cognition from a genetic perspective, and the map could be further expanded with the advanced phenotyping of human cognition and behavior traits. 


\section{Methods}

\section{WLS Study Population}

The WLS has measured 27,000+ phenotypic variables of 10,317 self-identified non-Hispanic

252 White individuals, who graduated from Wisconsin high schools in 1957, during 6 waves of data 253 collection over 60 years. The WLS also surveyed selected siblings and the spouse of the 254 original participants, expanding the total number of respondents to 19,050 individuals starting 255 from 1977. The cohort is representative of non-Hispanic White Americans who completed at 256 least 12 years of high school education in the United States.

Genotype data and Quality Control process of WLS In 2007-2008, saliva samples were collected by mail or by home-interview, and 9,019 individuals were successfully genotyped at the Johns Hopkins University center for inherited disease research (CIDR) using the Illumina HumanOmniExpress-24 v.1.1 array. The subsequent quality control process filtered individuals with (i) genotype missingness rate > 0.05 in all chromosomes, (ii) mismatch between recorded sex and genetically determined sex, (iii) high genetic relatedness with other individual ( $>0.025$ ), (iv) outlier in heterozygosity/homozygosity test, and (v) non-European ancestry outliers. Non-European individuals were identified by visually inspecting the principal component analysis (PCA) plot of the covariance matrix of the WLS genotype data with 1000 Genome populations. Additionally, SNPs with (i) genotype call rate $<0.95$, (ii) Hardy-Weinberg exact test $p$-value $<1.0 \mathrm{E}-05$, and (iii) minor allele frequency $<0.01$ were excluded from the data, resulting in 607,469 autosomal SNPs in 8,527 European-ancestry individuals. The data was then imputed to the Haplotype Reference Consortium (HRC) v1.1 European reference panel ${ }^{42}$ and resulted in 39,127,657 variants. The detailed imputation and QC report is available separately ${ }^{43,44}$.

\section{Cognitive GPS construction}

The WLS provided polygenic scores for four different cognitive traits based on GWAS MTAG summary statistics from Lee et al (REF), which were obtained from a multivariate analysis of educational attainment, cognitive performance, self-reported math ability and highest-level math class taken. GPS were calculated with PLINK 1.9 (REF) using the SNP weights adjusted 
279 for linkage disequilibrium using LDpred software (REF). All SNP weights were obtained from 280 cognitive GWAS discovery samples that did not contain the WLS participants (REF).

\section{Creation of the Cognitive Phenome}

283 1. Cognitive performance

284 The WLS administered seven types of cognitive modules over the survey periods, which were 285 used as the key components of the cognitive phenome: Similarities (administered in Round 286 4/5/6), Letter/Category Fluency (R5/6), Immediate/Delayed Word Recall (R5/6), Linguistic 287 Function (R5/6), Digit Ordering (R5/6), Number series (R6), and Health Literacy test scores. 288 Six of the seven cognition modules were repeatedly administered to the same participants. 289 More details of each module are illustrated in Supplementary Material.

290 2. Cognitive decline

291 We measured the degree of cognitive decline by comparing the two (three for similarity tests) 292 cognition test scores taken by the same individual, with time interval as the dependent variable 293 in the linear mixed model regression. We defined six measures of cognitive decline based on 294 the cognitive modules: similarities, letter fluency, category fluency, immediate recall, delayed 295 recall, and digit ordering.

3. Education

a. IQ score: All the respondents completed the Henmon-Nelson Test of Mental Ability with 90 items during their high school junior years (1956), which measured verbal, quantitative, and spatial knowledge $\mathrm{e}^{45-47}$. Raw scores were converted to IQ scores by standardizing to a mean of 100 based on Wisconsin centile rank (z-score).

b. Educational attainment: The years of education were estimated from the highest degree obtained by each participant, ranging from 12 to 20 years.

c. High School Class rank: Percentile rank information was retrieved from high school records, which is based on the mean grade taken throughout the high school courses. (100-(rank in class/(\# of students in class) $)^{*} 100$ ] The correlation between class grade and standardized test scores was as high as 0.90 , which reduces the possibility of teacher bias on grades or ranks ${ }^{48}$.

4. Personality 
309 WLS administered a short-version of the Big 5 Factor Model of Personality inventory test ${ }^{49}$ to

310 the respondents in the 1992-1993 collection wave: extraversion, openness, neuroticism,

311 conscientiousness, and agreeableness.

312 5. Leisure Activities

313 We investigated leisure activities of the WLS respondents to examine the genetic effects of

314 cognition regarding how time is allocated in individual habits, social roles, and behavioral

315 preferences. The contents of participants' leisure activities were self-reported in hours per

316 week and illustrated in the Supplementary Material. In order to correct for outliers with

317 extreme hours of certain activities, we took the natural logarithm of the reported hours for each

318 activity and ran linear regression with the built GPS.

319 6. Miscellaneous

320 a. Occupational Education Score: The WLS collected the first or only employment

321 information of the respondents. The measure assigned each employment with a numeric value for their industry or class-of-worker categories based on the 1990 US Census data, which represents a percentage of persons who had at least one year of college education, ranging from 0 to 999 .

b. Occupational Income Score: For the first or only job of the respondents in the employment modules, the WLS assigned the 1990-basis occupational earning scores, which represent the percentage of persons in the 1990 US Census data in an industry or class-of-work category who earned more than $\$ 14.30$ /hour in 1989 , ranging from 37 to 876 .

c. Spouse IQ: Previous literature has suggested the psychiatric hypothesis of assortative mating in academic achievements and IQ (i.e. individuals tend to select spouses similar in academic achievements and IQ to themselves) ${ }^{50-54}$. As spouse IQ data were available through the additional panels of WLS, we assessed the behavioral genetics of spouse concordance for IQ. We aimed to investigate if behaviors of assortative mating have pleiotropic associations with GPS of cognitive abilities.

\section{Phenome-wide Analysis}

Regression analyses mapped the four types of cognitive GPS (EA, CP, HM, MA) to the cognitive phenome that is created from the aforementioned normalized variables of the WLS 
340 data. For numerical phenotypes, linear regression is used to measure each GPS prediction 341 performance, adjusting for biological sex and the first 10 principal components (PC 1-10) of

342 ancestry. We measured each GPS' significance ( $p$-value), effect size ( $\beta$ ), 95\% confidence

343 interval $(\mathrm{Cl})$, and proportion of variance explained $\left(\mathrm{R}^{2}\right)$ on cognitive phenotype prediction. For 344 cognitive decline variables, we used linear mixed models, assuming fixed effects of each GPS

345 and covariates, and random effects for the weight level specific to each survey round and 346 individual. (c.f. WLS Round4 = timepoint 0, Round5= timepoint 1, Round6= timepoint 2) The 347 analyses were performed in R 3.5.1 environment, and linear mixed model was run with Ime4 348 package ${ }^{55}$. For each analysis, we excluded the respondents who had not completed all the 349 questionnaires for each phenotype. We used interaction $\mathrm{R}$ package to visualize our regression 350 results with interaction terms. 


\section{References.}

1 Deary, I. J. et al. Genetic contributions to stability and change in intelligence from childhood to old age. Nature 482, 212-215, doi:10.1038/nature10781 (2012).

2 Sharp, E. S. \& Gatz, M. Relationship between education and dementia: an updated systematic review. Alzheimer disease and associated disorders 25, 289-304, doi:10.1097/WAD.0b013e318211c83c (2011).

3 Andrews, S. J., Das, D., Cherbuin, N., Anstey, K. J. \& Easteal, S. Association of genetic risk factors with cognitive decline: the PATH through life project. Neurobiol Aging 41, 150-158, doi:10.1016/j.neurobiolaging.2016.02.016 (2016).

4 Morley, J. F. et al. Genetic influences on cognitive decline in Parkinson's disease. Mov Disord 27, 512-518, doi:10.1002/mds.24946 (2012).

5 Raj, T. et al. Genetic architecture of age-related cognitive decline in African Americans. Neurol Genet 3, e125, doi:10.1212/NXG.0000000000000125 (2017).

6 Furlong, L. I. Human diseases through the lens of network biology. Trends Genet 29, 150-159, doi:10.1016/j.tig.2012.11.004 (2013).

7 Hindorff, L. A. et al. Potential etiologic and functional implications of genome-wide association loci for human diseases and traits. Proc Natl Acad Sci U S A 106, 9362-9367, doi:10.1073/pnas.0903103106 (2009).

8 Chakravarti, A. \& Turner, T. N. Revealing rate-limiting steps in complex disease biology: The crucial importance of studying rare, extreme-phenotype families. Bioessays 38, 578-586, doi:10.1002/bies.201500203 (2016).

9 Lee, J. J. et al. Gene discovery and polygenic prediction from a genome-wide association study of educational attainment in 1.1 million individuals. Nat Genet 50, 1112-1121, doi:10.1038/s41588-018-0147-3 (2018).

10 Rietveld, C. A. et al. GWAS of 126,559 individuals identifies genetic variants associated with educational attainment. Science 340, 1467-1471, doi:10.1126/science.1235488 (2013).

11 Okbay, A. et al. Genome-wide association study identifies 74 loci associated with educational attainment. Nature 533, 539-542, doi:10.1038/nature17671 (2016).

12 Plomin, R. \& von Stumm, S. The new genetics of intelligence. Nat Rev Genet 19, 148-159, doi:10.1038/nrg.2017.104 (2018).

13 Trampush, J. W. et al. GWAS meta-analysis reveals novel loci and genetic correlates for general cognitive function: a report from the COGENT consortium. Mol Psychiatry 22, 1651-1652, doi:10.1038/mp.2017.197 (2017).

14 Sniekers, S. et al. Genome-wide association meta-analysis of 78,308 individuals identifies new loci and genes influencing human intelligence. Nat Genet 49, 1107-1112, doi:10.1038/ng.3869 (2017).

15 Davies, G. et al. Genome-wide association study of cognitive functions and educational attainment in UK Biobank ( $\mathrm{N}=112$ 151). Mol Psychiatry 21, 758-767, doi:10.1038/mp.2016.45 (2016).

16 Davies, G. et al. Genetic contributions to variation in general cognitive function: a meta-analysis of genome-wide association studies in the CHARGE consortium ( $\mathrm{N}=53949)$. Mol Psychiatry 20, 183-192, doi:10.1038/mp.2014.188 (2015). 
17 Davies, G. et al. Genome-wide association studies establish that human intelligence is highly heritable and polygenic. Molecular Psychiatry 16, 996-1005, doi:10.1038/mp.2011.85 (2011).

18 Bouchard, T. J., Jr. \& McGue, M. Genetic and environmental influences on human psychological differences. Journal of neurobiology 54, 4-45, doi:10.1002/neu.10160 (2003).

19 Herd, P., Carr, D. \& Roan, C. Cohort profile: Wisconsin longitudinal study (WLS). Int J Epidemiol 43, 34-41, doi:10.1093/ije/dys194 (2014).

20 Carney, A. K. Wisconsin longitudinal study. Int J Aging Hum Dev 79, 332-333, doi:10.1177/0091415015574179 (2014).

21 Wechsler, D. Wechsler adult intelligence scale-revised (WAIS-R). (Psychological Corporation, 1981).

22 Griffith, H. R. et al. A controlled quantitative MRI volumetric investigation of hippocampal contributions to immediate and delayed memory performance. J Clin Exp Neuropsychol 25, 1117-1127, doi:10.1076/jcen.25.8.1117.16731 (2003).

23 Golomb, J. et al. Hippocampal formation size in normal human aging: a correlate of delayed secondary memory performance. Learn Mem 1, 45-54 (1994).

24 Hackert, V. H. et al. Hippocampal head size associated with verbal memory performance in nondemented elderly. Neuroimage 17, 1365-1372, doi:10.1006/nimg.2002.1248 (2002).

25 Erickson, K. I. et al. Aerobic fitness is associated with hippocampal volume in elderly humans. Hippocampus 19, 1030-1039, doi:10.1002/hipo.20547 (2009).

26 Latimer, C. S. et al. Vitamin D prevents cognitive decline and enhances hippocampal synaptic function in aging rats. Proc Natl Acad Sci U S A 111, E4359-4366, doi:10.1073/pnas.1404477111 (2014).

27 Goveas, J. S. et al. Recovery of hippocampal network connectivity correlates with cognitive improvement in mild Alzheimer's disease patients treated with donepezil assessed by restingstate fMRI. J Magn Reson Imaging 34, 764-773, doi:10.1002/jmri.22662 (2011).

28 Jin, K. et al. Increased hippocampal neurogenesis in Alzheimer's disease. Proc Natl Acad Sci U S A 101, 343-347, doi:10.1073/pnas.2634794100 (2004).

29 Krishnan, K. R. et al. Randomized, placebo-controlled trial of the effects of donepezil on neuronal markers and hippocampal volumes in Alzheimer's disease. Am J Psychiatry 160, 20032011, doi:10.1176/appi.ajp.160.11.2003 (2003).

30 Toda, T. \& Gage, F. H. Review: adult neurogenesis contributes to hippocampal plasticity. Cell Tissue Res 373, 693-709, doi:10.1007/s00441-017-2735-4 (2018).

31 Snyder, J. S., Kee, N. \& Wojtowicz, J. M. Effects of adult neurogenesis on synaptic plasticity in the rat dentate gyrus. J Neurophysiol 85, 2423-2431, doi:10.1152/jn.2001.85.6.2423 (2001).

32 Scarmeas, N., Albert, S. M., Manly, J. J. \& Stern, Y. Education and rates of cognitive decline in incident Alzheimer's disease. J Neurol Neurosurg Psychiatry 77, 308-316, doi:10.1136/jnnp.2005.072306 (2006).

33 Stern, Y. Cognitive reserve in ageing and Alzheimer's disease. Lancet Neurol 11, 1006-1012, doi:10.1016/S1474-4422(12)70191-6 (2012).

34 Plassman, B. L. et al. Intelligence and education as predictors of cognitive state in late life: a 50year follow-up. Neurology 45, 1446-1450, doi:10.1212/wnl.45.8.1446 (1995).

35 Katzman, R. Education and the prevalence of dementia and Alzheimer's disease. Neurology 43, 13-20, doi:10.1212/wnl.43.1_part_1.13 (1993). 
36 Akshoomoff, N. et al. VIII. NIH Toolbox Cognition Battery (CB): composite scores of crystallized, fluid, and overall cognition. Monographs of the Society for Research in Child Development 78, 119-132, doi:10.1111/mono.12038 (2013).

37 Ackerman, P. L. \& Heggestad, E. D. Intelligence, personality, and interests: evidence for overlapping traits. Psychol Bull 121, 219-245, doi:10.1037/0033-2909.121.2.219 (1997).

38 DeYoung, C. G. in APA handbook of personality and social psychology, Volume 4: Personality processes and individual differences. APA handbooks in psychology ${ }^{\circledR}$. 369-399 (American Psychological Association, 2015).

39 DeYoung, C. G., Peterson, J. B. \& Higgins, D. M. Sources of openness/intellect: cognitive and neuropsychological correlates of the fifth factor of personality. Journal of personality 73, 825858, doi:10.1111/j.1467-6494.2005.00330.x (2005).

40 Schmidt, F. L. \& Hunter, J. General mental ability in the world of work: occupational attainment and job performance. J Pers Soc Psychol 86, 162-173, doi:10.1037/0022-3514.86.1.162 (2004).

41 Gottfredson, L. S. Why g matters: The complexity of everyday life. Intelligence 24, 79-132, doi:https://doi.org/10.1016/S0160-2896(97)90014-3 (1997).

42 McCarthy, S. et al. A reference panel of 64,976 haplotypes for genotype imputation. Nat Genet 48, 1279-1283, doi:10.1038/ng.3643 (2016).

43 Okbay, A., Benjamin, D. \& Visscher, P. Documentation for the data of Educational attainment, cognitive performance and math-related scores, $<$ https://www.ssc.wisc.edu/wlsresearch/documentation/GWAS/Lee et al (2018) PGS WLS.p df $>$ (2018).

44 Center, T. U. o. W. G. A. A Longitudinal Resource for Genetic Research in Behavioral and Health Sciences - Imputation Report (Wisconsin Longitudinal Study 2016).

45 Hauser, R. M. \& Palloni, A. Adolescent IQ and Survival in the Wisconsin Longitudinal Study. The Journals of Gerontology: Series B 66B, i91-i101, doi:10.1093/geronb/gbr037 \%J The Journals of Gerontology: Series B (2011).

46 Henmon, V. (Boston: Houghton-Mifflin Company, 1946).

47 Henmon, V. A. C. \& Holt, F. O. A Report on the Administration of Scholastic Aptitude Tests to 34,000 High School Seniors in Wisconsin in 1929 and 1930: Prepared for the Committee on Cooperation, Wisconsin Secondary Schools and Colleges. (Bureau of Guidance and Records of the University of Wisconsin, 1931).

48 Willingham, W. W., Pollack, J. M. \& Lewis, C. Grades and Test Scores: Accounting for Observed Differences. Journal of Educational Measurement 39, 1-37 (2002).

49 John, O. P., Donahue, E. M. \& Kentle, R. L. (Berkeley, CA: University of California, Berkeley, Institute of Personality ..., 1991).

50 Halpin, B. Educational homogamy in Ireland and Britain: trends and patterns. Br J Sociol 54, 473496, doi:10.1080/0007131032000143546 (2003).

51 Mascie-Taylor, C. G. \& Vandenberg, S. G. Assortative mating for IQ and personality due to propinquity and personal preference. Behav Genet 18, 339-345, doi:10.1007/bf01260934 (1988).

52 Watson, D. et al. Match makers and deal breakers: analyses of assortative mating in newlywed couples. Journal of personality 72, 1029-1068, doi:10.1111/j.0022-3506.2004.00289.x (2004).

53 Hur, Y. M. Assortive mating for personaltiy traits, educational level, religious affiliation, height, weight, adn body mass index in parents of Korean twin sample. Twin research : the official 
journal of the International Society for Twin Studies 6, 467-470, doi:10.1375/136905203322686446 (2003).

54 Pan, Y., Wang, K. Spousal concordance in academic achievements and IQ a principal component analysis. Open Journal of Psychiatry, 15-19, doi:10.4236/ojpsych.2011.12003 (2011).

55 Bates, D., Mächler, M., Bolker, B. \& Walker, S. Fitting Linear Mixed-Effects Models Using Ime4. 2015 67, Journal of Statistical Software, doi:10.18637/jss.v067.i01 (2015). 
Figure 1. Interaction plots showing different slopes of time-dependent interaction effects of cognitive GPS on the cognitive assessments that were administered to 8,507European ancestry individuals between the age of mid-60s (timepoint 0 ) and mid-70s (timepoint 1). Changes in five cognitive assessments (immediate recall test, category fluency test, digit ordering test, delayed recall test, and letter fluency test) and interaction effects of EA GPS (upper panel) and CP GPS (bottom panel) were shown. The X-axis indicates the timepoints of WLS survey round, while the $y$-axis indicates each cognitive assessment score. The three lines indicates different groups of individuals stratified by GPS. The gray area represents $95 \%$ confidence interval of each slope.

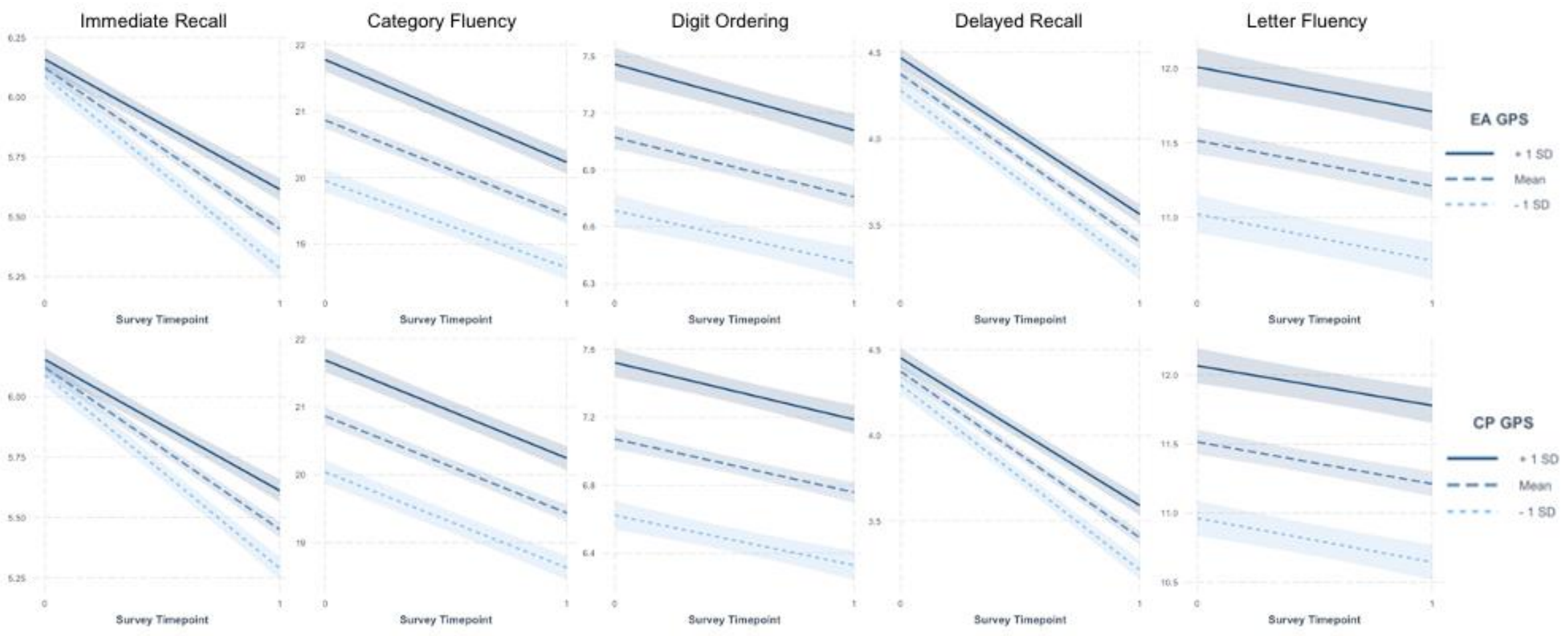


Figure 2. PheWAS plots of (a) Educational attainment (EA), and (b) Cognitive Performance (CP) GPS in the cognitive phenome of the WLS participants. The cognitive phenome on $x$-axis was created from the cognitive variables retrieved from WLS survey data, primarily from the cognition modules in the 2003-2005 and 2011-2013 waves. The red line represents the phenome-wide significance level [ $\log _{10}$ of the Bonferroni corrected $p$-value for multiple testing corrections (alpha $=0.05$ / $(58$ tested phenotypes $* 4$ GPS $)=2.15 \mathrm{E}-04)$.

(a) PheWAS plot of Educational Attainment (EA) GPS

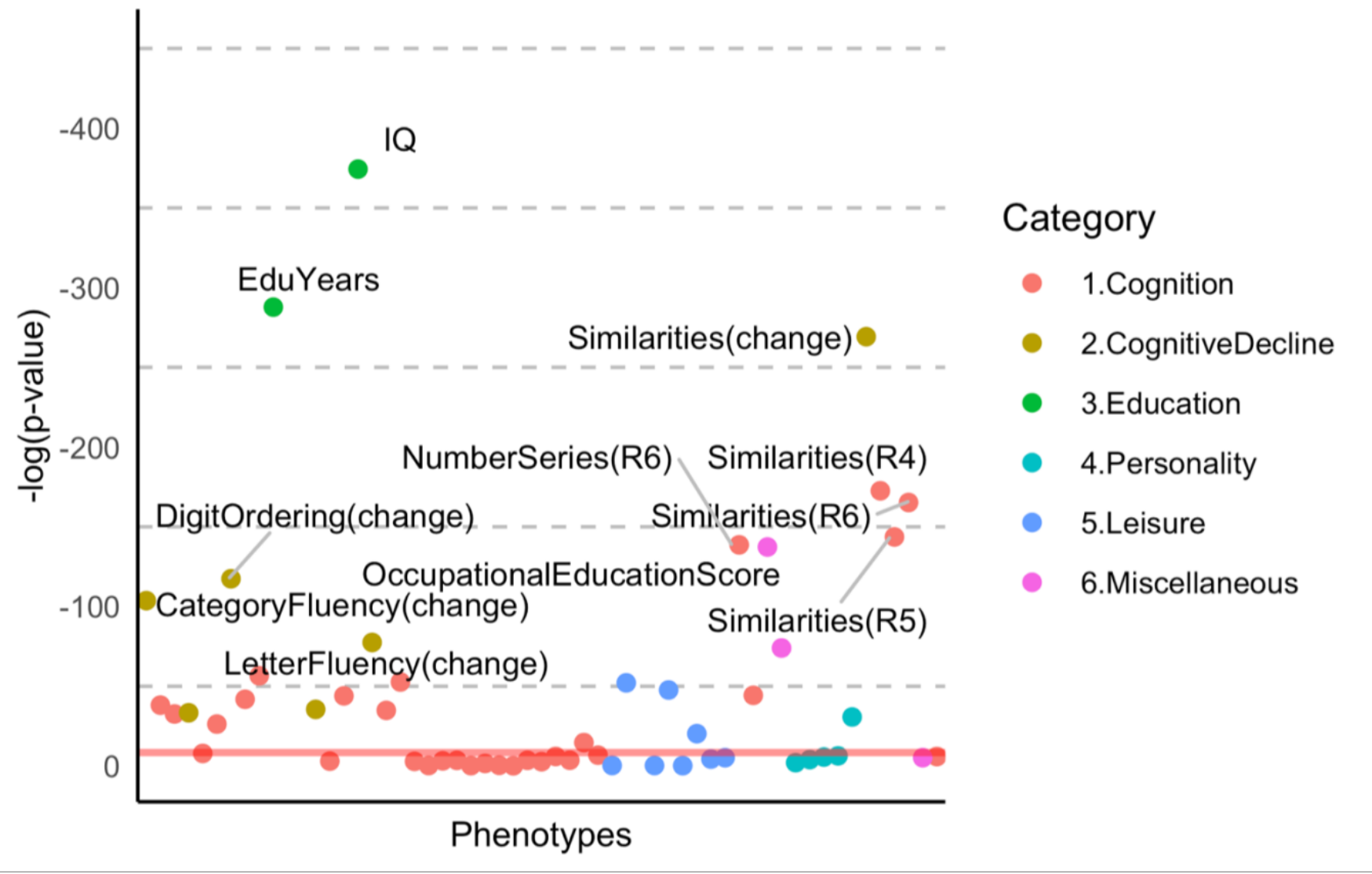


(b) PheWAS plot of Cognitive Performance (CP) GPS

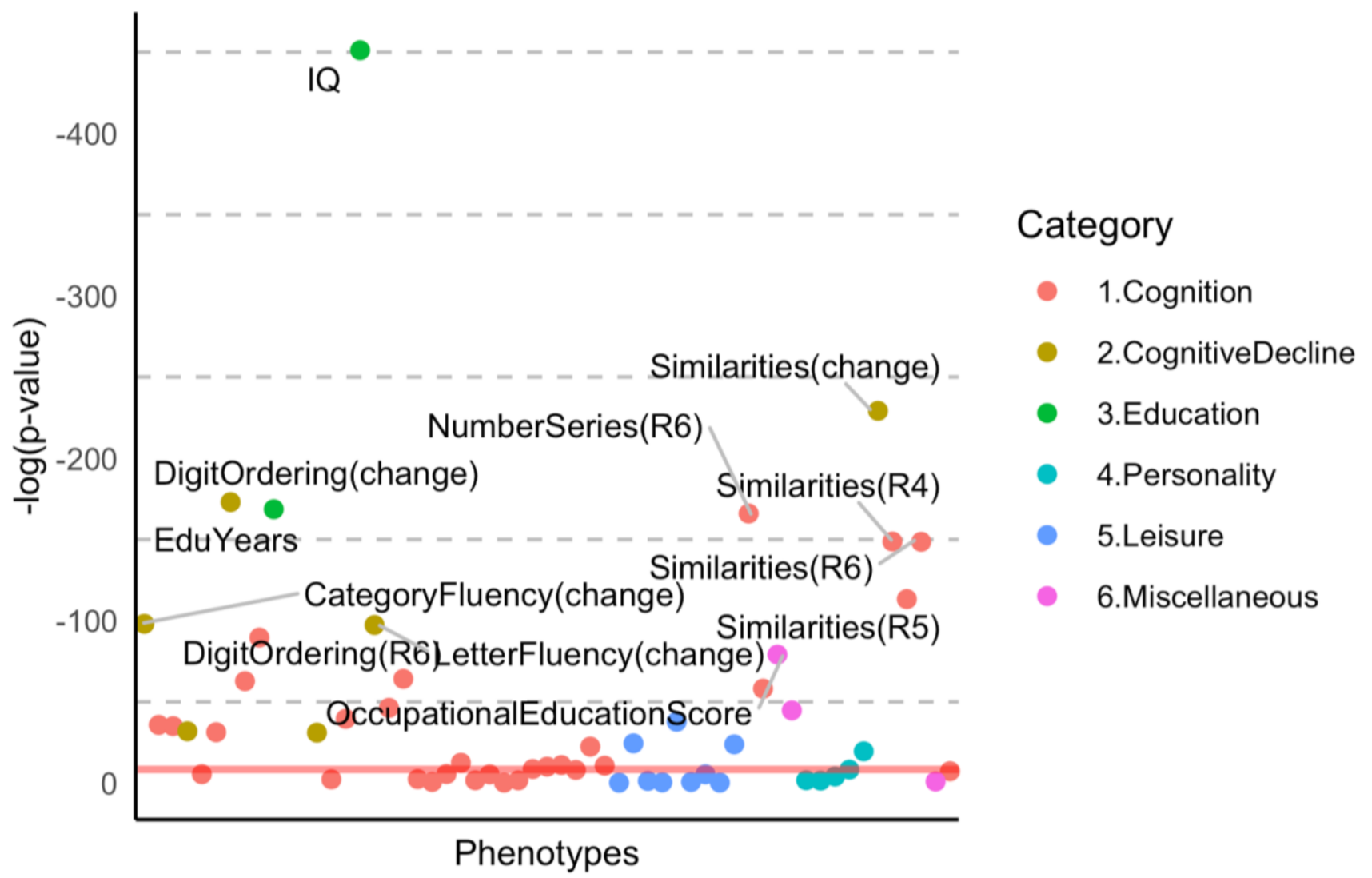




\section{Figure 3. Forest plots of the effect size $(\beta)$ of phenome-wide associations with GPS of (a)}

Educational attainment (EA), (b) Cognitive Performance (CP). Cognitive and behavioral phenotype variables are on the $y$-axis, and the number of asterisks $\left({ }^{*}\right)$ indicates the Bonferroni corrected significance of each phenome-wide association; * $p$-value of GPS-phenotype association $<0.05,{ }^{* *} p$ value of GPS-phenotype association $<5 \mathrm{E}-05,{ }^{* * *} \mathrm{p}$-value of GPS-phenotype association $<5 \mathrm{E}-07$. The numeric value of each effect size is specified as white-colored text in the colored circle, while the greycolored error bar around the circles indicates $95 \% \mathrm{Cl}$ of each effect size. 
(a) Forest plot of the effect sizes of PheWAS with GPS of Educational Attainment (EA)

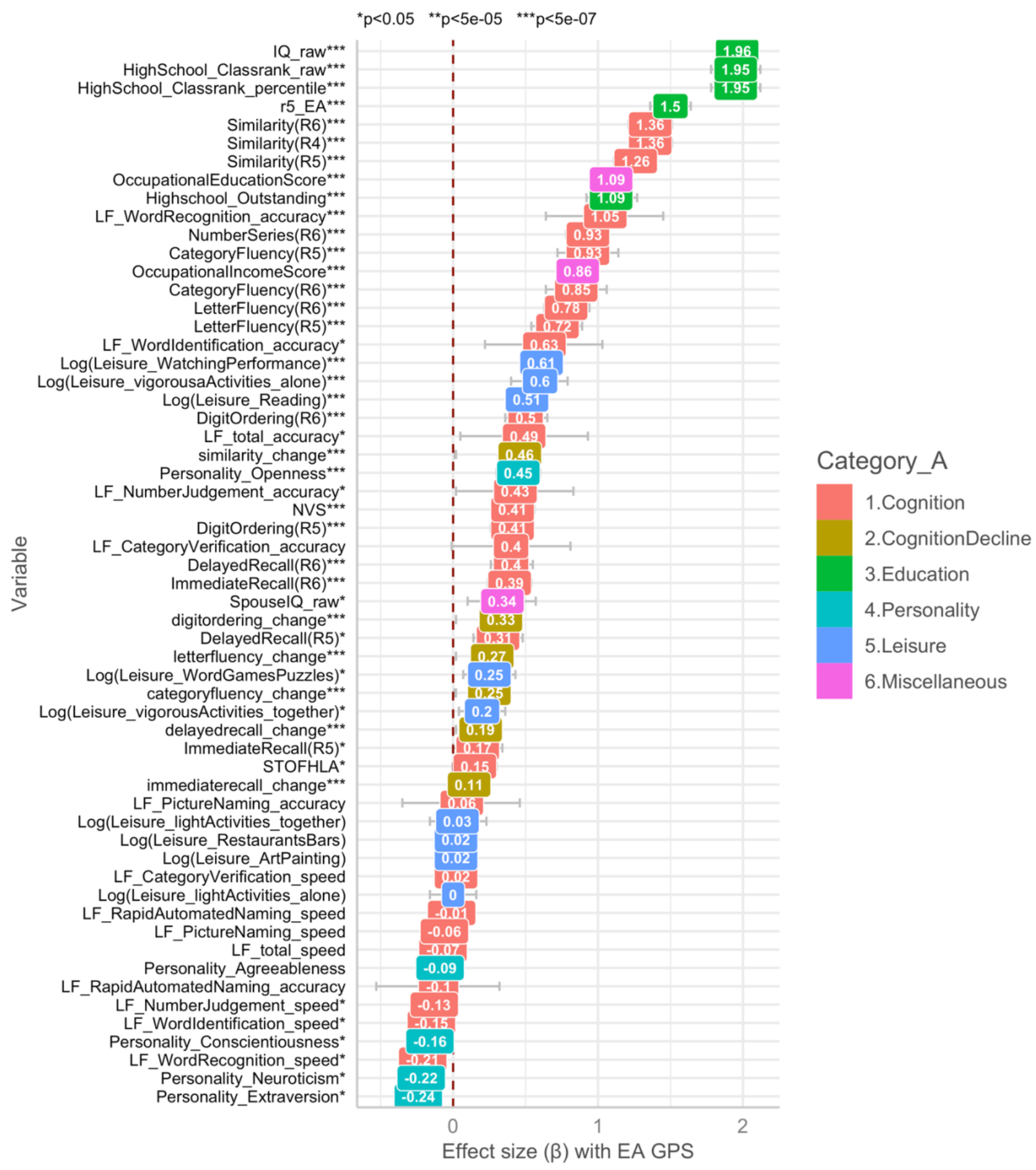




\section{(b) Forest plot of the effect sizes of PheWAS with GPS of Cognitive Performance (CP)}

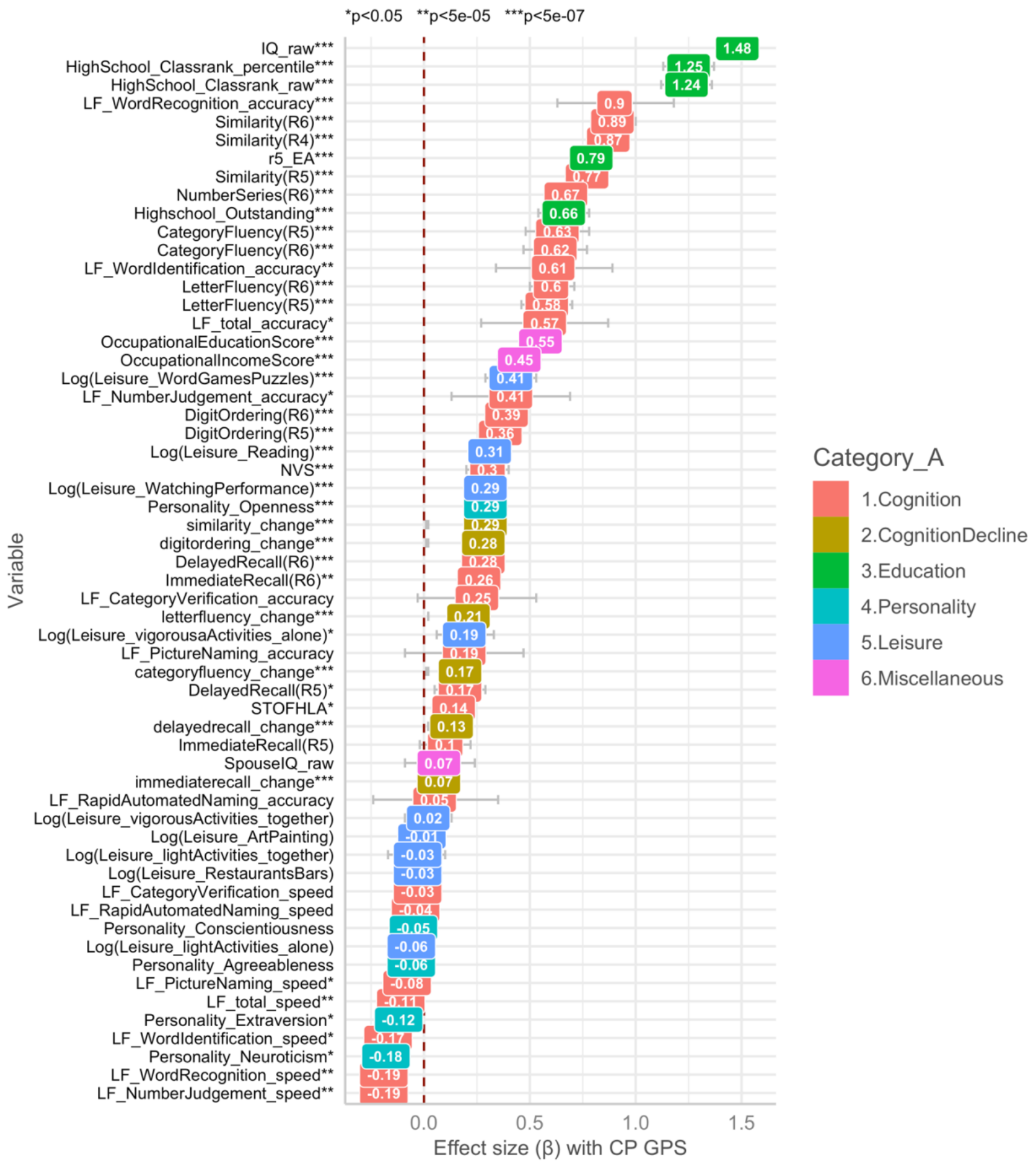


Table 1. PheWAS results of Educational attainment (EA) and Cognitive Performance (CP) GPS in the cognitive phenome of the WLS participants.

\begin{tabular}{|c|c|c|c|c|c|c|c|}
\hline & \multicolumn{3}{|c|}{ EA GPS } & \multicolumn{3}{|c|}{ CP GPS } \\
\hline & & $\beta$ & SE & $\mathrm{P}$ & & SE & $P$ \\
\hline \multirow{3}{*}{ Similarities } & time & -0.015 & 0.026 & $5.56 \mathrm{E}-01$ & -0.007 & 0.023 & 7.47E-01 \\
\hline & GPS & 3.152 & 0.160 & 1.67E-87 & 1.970 & 0.111 & 3.37E-71 \\
\hline & time $x$ & -0.063 & 0.080 & 4.36E-01 & -0.029 & 0.056 & $6.05 \mathrm{E}-01$ \\
\hline \multirow{3}{*}{ Delayed Recall } & time & -0.847 & 0.057 & $4.68 E-50$ & -0.801 & 0.049 & $1.41 \mathrm{E}-58$ \\
\hline & GPS & 0.609 & 0.136 & $7.50 \mathrm{E}-06$ & 0.353 & 0.094 & $1.79 \mathrm{E}-04$ \\
\hline & time $x$ GPS & 0.433 & 0.175 & $1.31 E-02$ & 0.498 & 0.122 & 4.22E-05 \\
\hline \multirow{3}{*}{ Immediate Recall } & time & -0.434 & 0.047 & $6.22 \mathrm{E}-20$ & -0.474 & 0.041 & $2.85 E-30$ \\
\hline & GPS & 0.236 & 0.112 & $3.49 \mathrm{E}-02$ & 0.146 & 0.078 & 5.96E-02 \\
\hline & time $x$ GPS & 0.834 & 0.146 & $1.22 E-08$ & 0.582 & 0.102 & $1.14 E-08$ \\
\hline \multirow{3}{*}{ Digit Ordering } & tin & -0.380 & 0.075 & 4.71E-07 & -0.346 & 0.066 & $1.43 \mathrm{E}-07$ \\
\hline & GP & 2.516 & 0.203 & 4.64E-35 & 2.032 & 0.140 & $3.47 \mathrm{E}-47$ \\
\hline & time $x$ GPS & -0.229 & 0.232 & 3.24E-01 & -0.092 & 0.162 & $5.68 \mathrm{E}-01$ \\
\hline \multirow{3}{*}{ Letter Fluency } & time & -0.291 & 0.088 & $9.27 \mathrm{E}-04$ & -0.279 & 0.077 & 2.62E-04 \\
\hline & GPS & 3.190 & 0.303 & $8.85 \mathrm{E}-26$ & 2.500 & 0.210 & $1.26 \mathrm{E}-32$ \\
\hline & time $x$ GPS & 0.045 & 0.271 & $8.69 \mathrm{E}-01$ & 0.071 & 0.189 & 7.06E-01 \\
\hline \multirow{3}{*}{ Category Fluency } & time & -1.646 & 0.127 & $3.22 E-38$ & -1.454 & 0.110 & $3.38 E-39$ \\
\hline & GF & 5.929 & 0.420 & $7.20 \mathrm{E}-45$ & 3.733 & 0.291 & $2.28 \mathrm{E}-37$ \\
\hline & time $\times$ GPS & -0.763 & 0.391 & $5.10 \mathrm{E}-02$ & -0.077 & 0.272 & 7.79E-01 \\
\hline
\end{tabular}


Table 2. Phenotypic variances $\left(R^{2}\right)$ explained by Educational attainment $(E A)$ and Cognitive Performance (CP) GPS in cognitive and behavioral phenomes of the WLS participants. Conditional $\mathrm{R}^{2}$ were reported, taking into account both the fixed and random effects, for the changes of cognitive test scores using linear mixed models. The numbers in parentheses indicate $\mathrm{R}^{2}$ adjusted for biological sex and the first 10 principal components of ancestry.

\begin{tabular}{|l|c|c|}
\hline Variable & $\begin{array}{c}\text { EA GPS } \\
\left.\mathbf{R}^{2} \text { (Adj. } \mathbf{R}^{2}\right)\end{array}$ & $\begin{array}{c}\text { CP GPS } \\
\left.\mathbf{R}^{2} \text { (Adj. } \mathbf{R}^{2}\right)\end{array}$ \\
\hline IQ & $0.098(0.096)$ & $0.115(0.114)$ \\
\hline Educational Attainment (R5) & $0.072(0.071)$ & $0.050(0.049)$ \\
\hline Similarities (change) & 0.543 & 0.543 \\
\hline Similarities test (R4) & $0.048(0.047)$ & $0.042(0.041)$ \\
\hline Similarities test (R5) & $0.041(0.040)$ & $0.033(0.032)$ \\
\hline Similarities test (R6) & $0.047(0.046)$ & $0.043(0.041)$ \\
\hline Letter Fluency (change) & 0.605 & 0.605 \\
\hline Letter Fluency test (R5) & $0.018(0.016)$ & $0.022(0.020)$ \\
\hline Letter Fluency test (R6) & $0.021(0.020)$ & $0.024(0.023)$ \\
\hline Category Fluency (change) & 0.578 & 0.578 \\
\hline Category Fluency test (R5) & $0.025(0.022)$ & $0.023(0.020)$ \\
\hline Category Fluency test (R6) & $0.022(0.019)$ & $0.024(0.021)$ \\
\hline Immediate Recall (change) & 0.177 & 0.177 \\
\hline Immediate Recall Test (R5) & $0.005(0.003)$ & $0.005(0.003)$ \\
\hline Immediate Recall Test (R6) & $0.006(0.005)$ & $0.006(0.005)$ \\
\hline Delayed Recall (change) & 0.216 & 0.217 \\
\hline Delayed Recall test (R5) & $0.004(0.002)$ & $0.003(0.001)$ \\
\hline Delayed Recall test (R6) & $0.006(0.005)$ & $0.006(0.005)$ \\
\hline Digit Ordering (change) & 0.347 & 0.347 \\
\hline Digit Ordering test (R6) & $0.009(0.008)$ & $0.010(0.009)$ \\
\hline Digit Ordering test (R5) & $0.008(0.006)$ & $0.010(0.009)$ \\
\hline Number series test (R6) & $0.023(0.021)$ & $0.025(0.023)$ \\
\hline NVS test (R6) & $0.005(0.004)$ & $0.006(0.004)$ \\
\hline STOFHLA test (R6) & $0.002(0.001)$ & $0.003(0.001)$ \\
\hline Linguistic Function - Speed (R6) & $0.014(0.003)$ & $0.027(0.016)$ \\
\hline Linguistic Function - Accuracy (R6) & $0.025(0.014)$ & $0.037(0.026)$ \\
\hline Spouse IQ & $0.006(0.003)$ & $0.004(0.000)$ \\
\hline Occupational Education Score & $0.036(0.035)$ & $0.024(0.023)$ \\
\hline Occupational Income Score & $0.021(0.020)$ & $0.014(0.013)$ \\
\hline Classrank in high school (percentile) & $0.092(0.090)$ & $0.080(0.078)$ \\
\hline Outstanding Student in high school & $0.035(0.033)$ & $0.028(0.026)$ \\
\hline & & \\
\hline
\end{tabular}


